\title{
Eine spieltheoretische Analyse von Zulieferer-Abnehmer-Beziehungen in Supply Chains
}

\author{
Eric Sucky \\ Aachen, 14. September 2001
}




\section{Gliederung}

1. Das Joint Economic-Lot-Size Model (JELS-Modell)

1.1 Individuell optimale Bestell- und Produktionspolitiken

1.2 Optimale gemeinsame Bestell- und Produktionspolitik

1.3 Vergleich der individuellen und gemeinsamen Bestell- und Produktionspolitiken

2. Verhandlungslösungen

2.1 Die Verhandlungssituation

2.2 Verhandlungslösungen bei vollständiger Information

3. Fazit und Ausblick 


\section{Das Joint Economic-Lot-Size Model (JELS-Modell) \\ 1.1 Individuell optimale Bestell- und Produktionspolitiken}

- Annahmen

- Es wird ein Abnehmer $(A)$ und ein Zulieferer $(P)$ eines bestimmten Gutes betrachtet.

- Der Abnehmer $(A)$ und der Zulieferer $(P)$ planen ihre individuell optimale Bestellmenge bzw. Losgröße mit Hilfe der klassischen Bestellmengenformel bzw. Losgrößenformel. 
- Planungssituation des Abnehmers (A)

- Der Gesamtbedarf je Periode beträgt b [ME/PE].

- Bei einer Periodendauer von $\tau$ Zeiteinheiten erfolgt der Lagerabgang kontinuierlich mit der konstanten Nachfragerate je Zeiteinheit von $\mathrm{b} / \tau$ [ME/ZE].

- Der Lagerzugang erfolgt ohne Lieferzeit (unendlich schnell) in gleich großen Losen von $x_{A}[M E]$.

- Fehlmengen sind nicht zugelassen (Bestellmenge=Liefermenge).

- Die Lagerkapazität ist ausreichend.

- Das Lager ist zu Beginn der Periode leer.

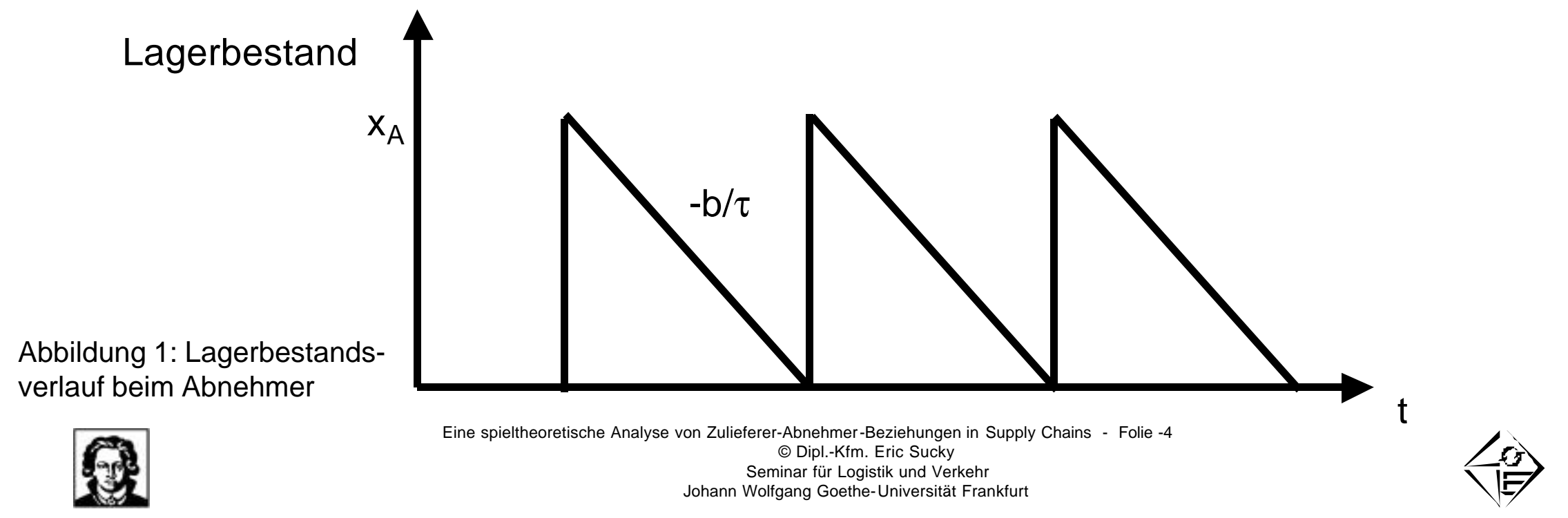




\section{- Individuell optimale Bestellpolitik des Abnehmers (A)}

- Die bestellfixen Kosten betragen B [GE].

- Die Lagerkosten betragen $\mathrm{h}_{\mathrm{A}}$ [GE/ME.PE].

- (A) bestimmt die Bestellmenge unter dem Ziel der Minimierung der entscheidungsrelevanten Kosten je Periode:

$$
\mathrm{K}^{\mathrm{A}}\left(\mathrm{x}_{\mathrm{A}}\right)=\mathrm{B} \cdot \frac{\mathrm{b}}{\mathrm{x}_{\mathrm{A}}}+\frac{\mathrm{x}_{\mathrm{A}}}{2} \cdot \mathrm{h}_{\mathrm{A}} \rightarrow \text { Min! }
$$

- Optimale Bestellmenge des Abnehmers $(A)$ :

$$
x_{A}^{*}=\sqrt{\frac{2 \cdot B \cdot b}{h_{A}}}
$$

- Minimale relevante Kosten des Abnehmers (A) je Periode:

$$
K^{A}\left(x_{A}^{*}\right)=\sqrt{2 \cdot B \cdot b \cdot h_{A}}
$$


- Planungssituation des Zulieferers (P)

- Die Gesamtnachfrage pro Periode beträgt b [ME/PE].

- Die Periodenkapazität beträgt d [ME/PE].

- Der Lagerzugang erfolgt mit einer konstanten Produktionsrate je Zeiteinheit (Produktionsgeschwindigkeit) $d / \tau$ [ME/ZE].

- Es gilt $d \geq b$ bzw. $d / \tau \geq b / \tau$. Nur so kann die Nachfrage befriedigt werden.

- Die Liefermenge entspricht dem Produktionslos $x_{P}[M E]$ („Lot-for-Lot Production Strategy“).

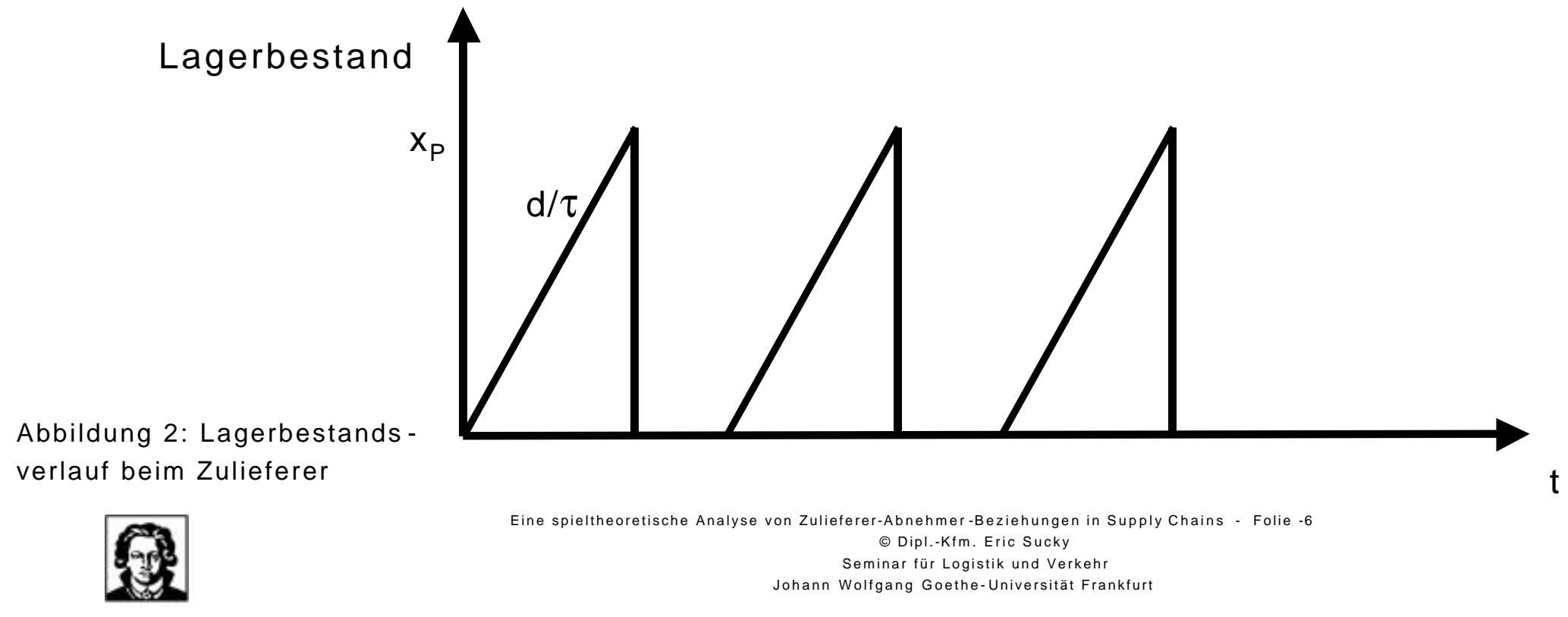


- Individuell optimale Produktionspolitik des Zulieferers (P)

- Die Rüstkosten betragen R [GE].

- Die Lagerkosten betragen $h_{P}$ [GE/ME.PE].

- $(\mathrm{P})$ bestimmt die Losgröße unter dem Ziel der Minimierung der entscheidungsrelevanten Kosten je Periode:

$$
\mathrm{K}^{\mathrm{P}}\left(\mathrm{x}_{\mathrm{P}}\right)=\mathrm{R} \cdot \frac{\mathrm{b}}{\mathrm{x}_{\mathrm{P}}}+\frac{\mathrm{x}_{\mathrm{P}}}{2} \cdot \frac{\mathrm{b}}{\mathrm{d}} \cdot \mathrm{h}_{\mathrm{p}} \rightarrow \text { Min! }
$$

- Optimale Losgröße des Zulieferers (P):

$$
x_{p}^{*}=\sqrt{\frac{2 \cdot R \cdot d}{h_{p}}}
$$

- Minimale relevante Kosten des Zulieferers $(P)$ je Periode:

$$
K^{P}\left(x_{p}^{*}\right)=b \cdot \sqrt{\frac{2 \cdot R \cdot h_{p}}{d}}
$$




\subsection{Optimale gemeinsame Bestell-und Produktionspolitik}

- Zur Ermittlung der gemeinsamen Bestell- und Produktionspolitik ist die Summe der relevanten Kosten des Abnehmers (A) und des Zulieferers $(P)$ je Periode zu minimieren:

$$
K^{G}\left(x_{G}\right)=\left(B \cdot \frac{b}{x_{G}}+\frac{x_{G}}{2} \cdot h_{A}\right)+\left(R \cdot \frac{b}{x_{G}}+\frac{x_{G}}{2} \cdot \frac{b}{d} \cdot h_{p}\right) \rightarrow \text { Min! }
$$

- Optimale gemeinsame Bestell- und Produktionspolitik:

$$
x_{G}^{*}=\sqrt{\frac{2 \cdot b \cdot(B+R)}{h_{A}+\frac{b}{d} \cdot h_{P}}}
$$

- Minimale entscheidungsrelevante Gesamtkosten je Periode:

$$
K\left(x_{G}^{*}\right)=\sqrt{2 \cdot b \cdot(B+R) \cdot\left(h_{A}+\frac{b}{d} \cdot h_{P}\right)}
$$


1.3 Vergleich der individuellen und gemeinsamen Bestell- und Produktionspolitiken

- Die Bestellmenge von $(A)$ muss dem Produktionslos von (P) entsprechen. Es gilt $x_{A}=x_{P}$.

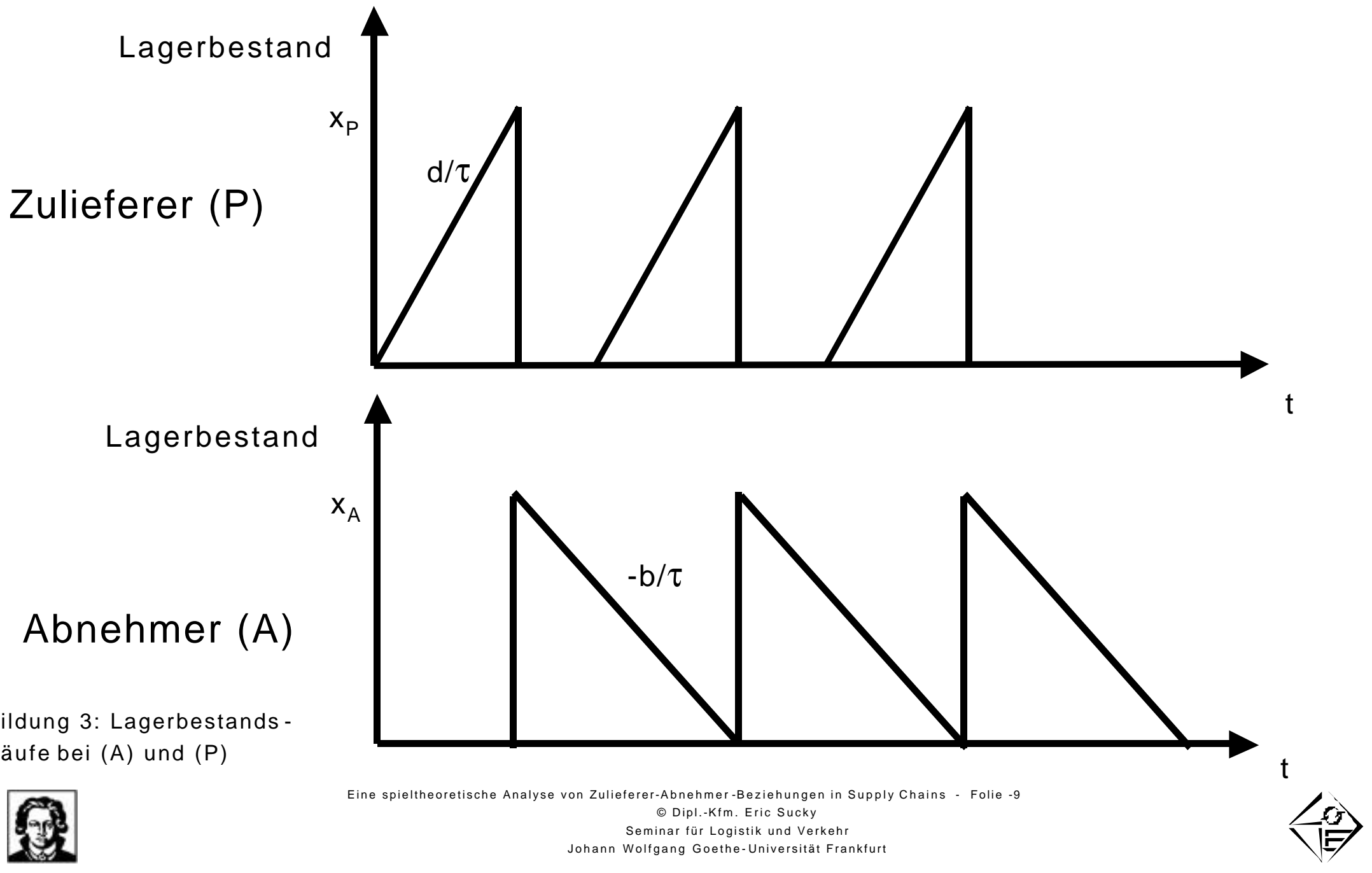


- Verhältnis der Rüstkosten von $(P)$ zu den Bestellkosten von (A) je Periode:

$$
\frac{K_{R}^{P}(x)}{K_{B}^{A}(x)}=\frac{R \cdot \frac{b}{x}}{B \cdot \frac{b}{x}}=\frac{R}{B}=\alpha
$$

mit $\alpha>0$

- Verhältnis der Lagerkosten von $(P)$ zu den Lagerkosten von (A) je Periode:

$$
\frac{K_{L}^{P}(x)}{K_{L}^{A}(x)}=\frac{\frac{x}{2} \cdot \frac{b}{d} \cdot h_{p}}{\frac{x}{2} \cdot h_{a}}=\frac{b \cdot h_{P}}{d \cdot h_{A}}=\beta
$$

mit $\beta>0$ 
- Beziehungen zwischen den Bestell- und Produktionspolitiken:

$$
\begin{array}{ll}
x_{A}^{*}=\sqrt{\frac{\beta}{\alpha}} \cdot x_{P}^{*} & x_{P}^{*}=\sqrt{\frac{\alpha}{\beta}} \cdot x_{A}^{*} \\
x_{G}^{*}=\sqrt{\frac{1+\alpha}{1+\beta}} \cdot x_{A}^{*} & x_{G}^{*}=\sqrt{\frac{1+\frac{1}{\alpha}}{1+\frac{1}{\beta}}} \cdot x_{P}^{*}
\end{array}
$$

- Nur für $\alpha=\beta$ ist $x_{A}{ }^{*}=x_{P}{ }^{*}=x_{G}{ }^{*}$.

- Für $\alpha \neq \beta$ gilt :

$$
\left.\mathrm{x}_{\mathrm{G}}^{*} \in\right] \mathrm{x}_{\mathrm{A}}^{*}, \mathrm{x}_{\mathrm{P}}^{*}[
$$




\section{Zwischenergebnisse (1)}

- Die Bestellmenge von (A) muss dem Produktionslos von (P) entsprechen. Es gilt $x_{G}=x_{A}=x_{P}$.

- Für den Fall $\alpha=\beta$ gilt $x^{*}{ }_{G}=x^{*}{ }_{A}=x^{*}{ }_{P}$.

- Für den Fall $\alpha \neq \beta$ existiert im dargestellten Modell nur dann eine Lösung, wenn

- (P) sich an die optimale Bestellmenge von (A) anpasst $\left(\mathrm{X}_{\mathrm{G}}=\mathrm{X}_{\mathrm{A}}{ }_{\mathrm{A}}=\mathrm{X}_{\mathrm{P}}\right)$,

- (A) sich an die optimale Losgröße von (P) anpasst $\left(x_{G}=x_{A}=x_{P}^{*}\right)$ oder

- (A) und (P) sich auf eine andere gemeinsame Bestell- und Produktionspolitik einigen, z.B. die Realisierung der optimalen gemeinsamen Bestell- und Produktionspolitik $\left(\mathrm{X}^{*}{ }_{\mathrm{G}}=\mathrm{X}_{\mathrm{A}}=\mathrm{X}_{\mathrm{P}}\right)$. 


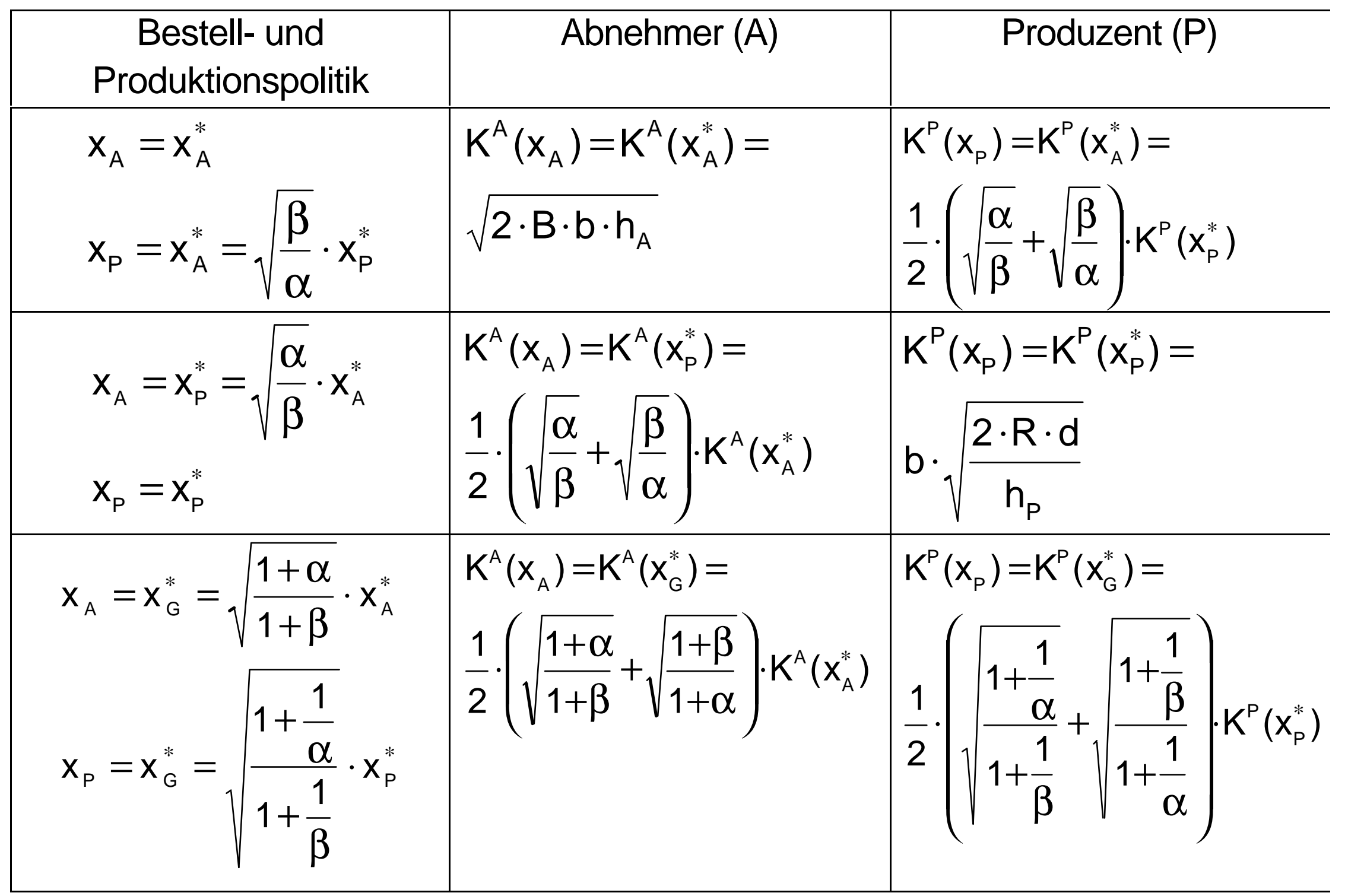

Tabelle 1: Kostenwirkungen alternativer Bestell- und Produktionspolitiken (vgl. Toporowski, 1996) 
- Für $\alpha \neq \beta, \alpha, \beta>0$ gilt:

$$
\begin{aligned}
& \frac{1}{2} \cdot\left(\sqrt{\frac{\alpha}{\beta}}+\sqrt{\frac{\beta}{\alpha}}\right)>1 \quad \frac{1}{2} \cdot\left(\sqrt{\frac{1+\alpha}{1+\beta}}+\sqrt{\frac{1+\beta}{1+\alpha}}\right)>1 \quad \frac{1}{2} \cdot\left(\sqrt{\frac{1+\frac{1}{\alpha}}{1+\frac{1}{\beta}}}+\sqrt{\frac{1+\frac{1}{\beta}}{1+\frac{1}{\alpha}}}\right)>1 \\
& \frac{1}{2} \cdot\left(\sqrt{\frac{\alpha}{\beta}}+\sqrt{\frac{\beta}{\alpha}}\right)>\frac{1}{2} \cdot\left(\sqrt{\frac{1+\alpha}{1+\beta}}+\sqrt{\frac{1+\beta}{1+\alpha}}\right) \\
& \frac{1}{2} \cdot\left(\sqrt{\frac{\alpha}{\beta}}+\sqrt{\frac{\beta}{\alpha}}\right)>\frac{1}{2} \cdot\left(\sqrt{\frac{1+\frac{1}{\alpha}}{1+\frac{1}{\beta}}}+\sqrt{\frac{1+\frac{1}{\beta}}{1+\frac{1}{\alpha}}}\right)
\end{aligned}
$$


Zwischenergebnisse (2)

- Ein Abweichen von der individuell optimalen Bestell- bzw. Produktionspolitik ist immer mit einem Anstieg der relevanten Kosten des Abweichenden verbunden.

- Verhalten sich $(A)$ und $(P)$ rational im Sinne der verfolgten Zielsetzungen, so wählen sie ihre jeweils individuell optimale Bestell- bzw. Produktionspolitik $x^{*}{ }_{A}$ bzw. $x^{*}{ }_{P}$.

- Für den Fall $\alpha \neq \beta$ existiert im JELS-Modell keine Lösung, da nur für $\alpha=\beta$ auch $x^{*}{ }_{G}=x^{*}{ }_{A}=x^{*}{ }_{P}$ gilt.

- Für den Fall $\alpha \neq \beta$ kann eine gemeinsame Bestell- und Produktionspolitik nur aus Verhandlungen zwischen $(A)$ und $(\mathrm{P})$ resultieren. 


\section{Verhandlungslösungen \\ 2.1 Die Verhandlungssituation}

- (A) verfügt über die Marktmacht, seine individuell optimale Bestellmenge $\mathrm{x}_{\mathrm{A}}{ }_{\mathrm{A}}$ gegenüber $(\mathrm{P})$ durchzusetzen.

- Für $(A)$ besteht kein Anreiz, von dieser individuell optimalen Bestellpolitik abzuweichen.

- Ohne Verhandlungen wird $x^{*}{ }_{A}$ realisiert:

$$
\mathrm{K}^{\mathrm{A}}\left(\mathrm{x}_{\mathrm{A}}^{*}\right)=\sqrt{2 \cdot \mathrm{B} \cdot \mathrm{b} \cdot \mathrm{h}_{\mathrm{A}}} \quad \mathrm{K}^{\mathrm{P}}\left(\mathrm{x}_{\mathrm{A}}^{*}\right)=\frac{1}{2} \cdot\left(\sqrt{\frac{\alpha}{\beta}}+\sqrt{\frac{\beta}{\alpha}}\right) \cdot \mathrm{K}^{\mathrm{P}}\left(\mathrm{x}_{\mathrm{P}}^{*}\right)
$$


- Damit $(A)$ eine andere Menge als $x^{*}{ }_{A}$ wählt, muss mindestens der daraus resultierende Kostenanstieg bei $(A)$ durch Zahlungen von $(P)$ an $(A)$ kompensiert werden.

- (P) kann versuchen, (A) durch Kompensationszahlungen zur Wahl einer anderen als dessen individuell optimalen Bestellpolitik zu bewegen.

- (P) möchte (A) das Angebot unterbreiten, eine gemeinsame Bestell- und Produktionspolitik $x_{G}$ gegen eine Kompensationszahlung in Höhe von z zu wählen. 
- Es liegt ein Ultimatumsspiel vor, d.h. (P) macht ein „take-it-orleave-it"-Angebot. Das Spiel ist sofort nach Annahme oder Ablehnung durch $(A)$ beendet.

- Es entstehen keine Transaktionskosten, z.B. Informationskosten oder Verhandlungskosten.

- Welche Bestell- und Produktionspolitik $x_{G}=x_{A}=x_{P}$ ergibt sich als Verhandlungslösung? 


\subsection{Verhandlungslösungen bei vollständiger Information}

- (P) verfügt über vollständige Informationen bezüglich der Funktion der relevanten Kosten von $(A)$.

- Das Optimierungsproblem von $(P)$ lautet:

$\min \mathrm{K}^{\mathrm{P}}\left(\mathrm{x}_{\mathrm{G}}, \mathrm{z}\right)=\mathrm{K}^{\mathrm{P}}\left(\mathrm{x}_{\mathrm{G}}\right)+\mathrm{z}$

u.d.N.

$\mathrm{K}^{\mathrm{A}}\left(\mathrm{x}_{\mathrm{A}}^{*}\right)-\mathrm{K}^{\mathrm{A}}\left(\mathrm{x}_{\mathrm{G}}\right)+\mathrm{Z} \geq 0 \quad$ Teilnahmebedingung

$x_{G}, z \geq 0$ 
- Karush-Kuhn-Tucker-Bedingungen

$$
\begin{aligned}
& \frac{\partial L}{\partial x_{G}}=\frac{\partial K^{P}\left(x_{G}\right)}{\partial x_{G}}+\lambda \cdot \frac{\partial K^{A}\left(x_{G}\right)}{\partial x_{G}} \geq 0 \\
& x_{G} \cdot\left(\frac{\partial K^{P}\left(x_{G}\right)}{\partial x_{G}}+\lambda \cdot \frac{\partial K^{A}\left(x_{G}\right)}{\partial x_{G}}\right)=0 \\
& \frac{\partial L}{\partial z}=1-\lambda \geq 0 \\
& z \cdot(1-\lambda)=0 \\
& -\frac{\partial L}{\partial \lambda}=K^{A}\left(x_{A}^{*}\right)-K^{A}\left(x_{G}\right)+z \geq 0 \\
& \lambda \cdot\left(K^{A}\left(x_{A}^{*}\right)-K^{A}\left(x_{G}\right)+z\right)=0 \\
& x_{G}, z, \lambda \geq 0
\end{aligned}
$$


- Für $z>0$ folgt: $\lambda=1$

- Mit $\lambda=1$ und $x_{G}>0$ ergibt sich:

$$
\begin{aligned}
& \frac{\partial K^{P}\left(x_{G}\right)}{\partial x_{G}}+\frac{\partial K^{A}\left(x_{G}\right)}{\partial x_{G}}=0 \\
& z=K^{A}\left(x_{G}\right)-K^{A}\left(x_{A}^{*}\right)
\end{aligned}
$$


- (P) wählt genau die Menge $X^{*}{ }_{G}$, die sich bei einer gemeinsamen Festlegung der Bestell- und Produktionspolitik im JELS-Modell ergibt:

$$
x_{G}^{*}=\sqrt{\frac{2 \cdot b \cdot(B+R)}{h_{A}+\frac{b}{d} \cdot h_{P}}}
$$

- Die angebotene Kompensationszahlung von $(P)$ an $(A)$ beträgt:

$$
\begin{aligned}
z\left(x_{G}^{*}\right) & =K^{A}\left(x_{G}^{*}\right)-K^{A}\left(x_{A}^{*}\right) \\
& =\left[\frac{1}{2} \cdot\left(\sqrt{\frac{1+\alpha}{1+\beta}}+\sqrt{\frac{1+\beta}{1+\alpha}}\right) \cdot \sqrt{2 \cdot B \cdot b \cdot h_{A}}\right]-\sqrt{2 \cdot B \cdot b \cdot h_{A}}
\end{aligned}
$$

- Verhält sich $(A)$ rational im Sinne seiner Zielsetzung, so nimmt er das Angebot an, da er nicht schlechter gestellt wird als bei Wahl seiner individuell optimalen Bestellmenge $\mathrm{x}^{*}{ }_{\mathrm{A}}$. 
- Lohnt sich dieses Angebot für $(P)$ ?

- Damit sich das Angebot für (P) lohnt, muss gelten:

$$
\begin{aligned}
& K^{P}\left(x_{G}^{*}\right)+z<K^{P}\left(x_{A}^{*}\right) \Leftrightarrow \\
& K^{P}\left(x_{G}^{*}\right)+K^{A}\left(x_{G}^{*}\right)<K^{P}\left(x_{A}^{*}\right)+K^{A}\left(x_{A}^{*}\right) \Leftrightarrow
\end{aligned}
$$$$
\frac{1}{2} \cdot\left(\sqrt{\frac{1+\frac{1}{\alpha}}{1+\frac{1}{\beta}}}+\sqrt{\frac{1+\frac{1}{\beta}}{1+\frac{1}{\alpha}}}\right) \cdot K^{P}\left(x_{P}^{*}\right)+\frac{1}{2} \cdot\left(\sqrt{\frac{1+\alpha}{1+\beta}}+\sqrt{\frac{1+\beta}{1+\alpha}}\right) \cdot K^{A}\left(x_{A}^{*}\right)
$$$$
<\frac{1}{2} \cdot\left(\sqrt{\frac{\alpha}{\beta}}+\sqrt{\frac{\beta}{\alpha}}\right) \cdot K^{\mathrm{P}}\left(\mathrm{x}_{\mathrm{P}}^{*}\right)+\mathrm{K}^{\mathrm{A}}\left(\mathrm{x}_{\mathrm{A}}^{*}\right)
$$ 


$$
\frac{\frac{1}{2} \cdot\left(\sqrt{\frac{1+\frac{1}{\alpha}}{1+\frac{1}{\beta}}}+\sqrt{\frac{1+\frac{1}{\beta}}{1+\frac{1}{\alpha}}}\right)+\frac{1}{2} \cdot\left(\sqrt{\frac{1+\alpha}{1+\beta}}+\sqrt{\frac{1+\beta}{1+\alpha}}\right) \cdot \frac{1}{\sqrt{\alpha \cdot \beta}}-\frac{1}{\sqrt{\alpha \cdot \beta}}}{\frac{1}{2} \cdot\left(\sqrt{\frac{\alpha}{\beta}}+\sqrt{\frac{\beta}{\alpha}}\right)}<1
$$

- Das Angebot lohnt sich für den Produzenten $(P)$, da die Bedingung für alle $\alpha, \beta>0$, mit $\alpha \neq \beta$, erfüllt ist.

- (P) realisiert einen Verhandlungsgewinn in Höhe von:

$$
V^{P}\left(x_{G}^{*}\right)=K^{P}\left(x_{A}^{*}\right)-\left(K^{P}\left(x_{G}^{*}\right)+z\left(x_{G}^{*}\right)\right)>0
$$




\section{Fazit und Ausblick}

- Verhandlungslösungen bei vollständiger Information

- $(\mathrm{P})$ verhält sich wie eine zentrale Planungsinstanz und wählt genau die Menge $\mathrm{X}^{*}{ }_{\mathrm{G}}$, die sich bei gemeinsamer Festlegung der Bestell- und Produktionspolitik im JELS-Modell ergibt.

- Verhält $(A)$ sich rational im Sinne seiner Zielsetzung, so nimmt er das Angebot an, da er nicht schlechter gestellt wird als bei Realisierung seiner individuell optimalen Bestellmenge.

- (P) erhält den gesamten Verhandlungsgewinn.

- Verhandlungen führen bei vollständiger Information genau zur gemeinsamen, optimalen Bestell- und Produktionspolitik $\mathrm{X}_{\mathrm{G}}{ }_{\mathrm{G}}$, unabhängig davon, wer die Marktmacht besitzt seine individuell optimale Menge durchzusetzen und auch unabhängig davon wer das Angebot abgibt. 
- Weiterführende Analysen:

- Analyse des Verhandlungsprozesses bei unvollständiger Information.

- Analyse des Verhandlungsprozesses bei mehrstufigen Spielen.

- Analyse der Zulieferer-Abnehmer-Beziehungen, für den Fall, dass der Produzent keine "Lot-for-Lot Production Strategy“ verfolgt.

- Analyse der Zulieferer-Abnehmer-Beziehungen, für den Fall, dass Produzent und Abnehmer dynamische Modelle zur Bestell- und Losgrößenplanung verwenden. 